\title{
Online collection of Patient Reported Outcome Measures: an effective method for follow-up of benign surgery
}

\author{
Jan H Koetje MD PhD ${ }^{1 *}$, Gooitzen M Van Dam MD PhD ${ }^{2}$, Joep Dille ${ }^{3}$ and Vincent B Nieuwenhuijs MD PhD ${ }^{1}$ \\ ${ }^{1}$ Isala, Department of Surgery, Dr. Van Heesweg 2, 8025 AB, Zwolle, The Netherlands \\ ${ }^{2}$ University Medical Center Groningen, Department of Surgery, Hanzeplein 1, Groningen, The Netherlands \\ ${ }^{3}$ Isala Academy, Innovation and Science, Dr. Van Deenweg 1, 8025 BP Zwolle, Zwolle, The Netherlands
}

\begin{abstract}
Background: Long-term follow-up for benign surgery is of importance, but due to small administrative and logistic capacity not available. This study describes the results of our online data collection system used in a cohort of patients after antireflux surgery. We compared the online system with the previously used paper based data collection.

Methods: This study included 294 patients following laparoscopic antireflux surgery. It compares response rates and completeness of collected data between a group that uses an online data collection system, using automated e-mails, and a group that completed data on paper. Also, improvement and satisfaction after surgery are compared between both methods of data collection.

Results: Response rates with online data collection were $86.3 \%-90.4 \%$. This is significant higher when compared to collection with paper questionnaires (11.9\%$66.4 \%$; $\mathrm{P}<0.001)$. Completeness of collected data is higher with the online system as well (94.9\%-99.3\% vs. 54.5\%-75.0\%; $\mathrm{P}<0.05)$. Improvement of GORD-related quality of life, improvement of dysphagia complaints, satisfaction of surgery and the number of patients that would undergo this operation again (based on their postoperative situation) are equal in the online and paper group.

Conclusions: Online follow-up leads to high response rates and high completeness of data. It is a user-friendly way to collect data in large cohorts of patient with benign pathology. This data can be used to inform patients on the surgeon or hospital results for a specific treatment and is useful for healthcare providers to evaluate treatments.
\end{abstract}

\section{Introduction}

Patient Reported Outcome Measures (PROMs) are increasingly used as valuable parameters of quality of medical care [1-4]. The main advantage of PROMs is that the patient's opinion is not interpreted or influenced by a researcher or clinician [5].

For malignant diseases, data collection and evaluation of care is an integrated and established part of clinical practice. Funded by health care companies, it is well-organized, validated and reliable according to (inter-) national guidelines and databases [6-13]. However, for benign pathology, follow-up after treatment is often not available.

In benign surgery the balance of surgical harm and change in quality of life is more important, since surgery is not lifesaving. The outcomes of treatments could be registered, amongst others to inform other patients. The increasing demand of clinical results in health care by National Health Agencies and insurance companies to justify hospital contracts may be another interesting reason to report outcome of treatment in benign disease [14].

Active follow-up after benign surgery carries a considerable high logistic and administrative burden, making it often impossible to follow patients yearly, notwithstanding the impact on costs of data managers and hospital visits. For this reason, an unmet need exists for alternative methods for follow-up. As a consequence, we have attempted to provide potential solutions for this problem.

In our practice, we have chosen to use routine questionnaires, printed on paper, and handed these to the patients during their visit to the outpatient clinic before and after laparoscopic antireflux surgery or sent the questionnaires by regular mail. These data were collected prospectively for all patients on yearly base, and consisted of questionnaires, both validated health-related, quality of life and disease specific as well as questions to evaluate quality of care. Since not all questionnaires were completed by all patients and therefore often not useable, we were searching for a better follow-up method. In our hospital an online data collection system was developed (Research Manager) with an option for online questioning per e-mail. We hypothesised that this online follow-up would lead to a better response rate and more complete data. In this study we describe the response rate and completeness of the collected data between the online and 'offline' questionnaires in a large cohort of patients after laparoscopic antireflux surgery.

\section{Material and methods}

All patients that were planned for antireflux surgery suffering gastro-oesophageal reflux disease (GORD), hiatal hernia oeophagei

Correspondence to: JH Koetje, Isala Zwolle, Department of Surgery, Postbus 10400, 8000 GK Zwolle, The Netherlands, Tel: +31-38-4248265; Fax: +31-384247720; E-mail: koetje@isala.nl

Key words: patient reported outcome measures, online data collection, laparoscopic antireflux surgery

Received: January 19, 2018; Accepted: January 28, 2018; Published: January 30, 2018 
(HHO) or achalasia in our hospital were asked to join the follow-up program. Patients who were mentally incapable to fill in questionnaires, younger than 18 years, incapable to speak the Dutch language or patients that were diagnosed for a different disease during preoperative investigations or during surgery were excluded. QoL measurements consisted of one preoperative questionnaire, and six postoperative questionnaires. The first postoperative questionnaire was send three months postoperative, the following questionnaires were send one year up until five years postoperative.

Patients were seen in the outpatient clinic four weeks and three months postoperative, and again if needed. All patients received yearly telephone calls up until five years postoperatively.

\section{Surgical procedures}

All surgical procedures were performed laparoscopically. Fundoplications were performed in three different methods. The most used is the $180^{\circ}$ anterior fundoplication as described by Gatenby, et al [15]. The laparoscopic $360^{\circ}$ Nissen fundoplication has been described by Jamieson, et al [16]. The Toupet fundoplication is a partial posterior $\left(270^{\circ}\right)$ fundoplication [17]. We performed this type of fundoplication mostly in 2013 and 2014 in a multicenter trial that compared the $180^{\circ}$ anterior fundoplication with the Toupet fundoplication (MANTA trial).

For patients suffering achalasia we performed a laparoscopic Heller myotomy [18], followed by a $180^{\circ}$ anterior Dor fundoplication, as described by Rosati [19].

\section{Objective follow-up}

Barium-swallow X-rays were performed three to six months postoperative. Objective investigations such as 24-hours-pH-metric, endoscopy, oesophageal manometry were performed postoperatively only on indication given the invasive character.

\section{Questionnaires}

The questionnaires included the validated Gastro-Esophageal Reflux Disease health related Quality of Life (GERD-hr-QoL) for GERD-related quality of life [20-23], and the validated QLQ-OES-24 for scoring dysphagia [24,25]. We used 10-point Visual Analogue Scales [26], and asked patients to score their symptoms, satisfaction about preoperative information, quality of care (outpatient, surgical department, ward, etc), waiting time for the operation, postoperative care, and satisfaction after surgery. Also, we asked patients if they would undergo the same operation again, should have undergone this operation earlier and if they would recommend this operation to a close relative or good friend with equal symptoms, knowing what the results are after surgery. All questionnaires were given at all time points.

\section{Follow-up on paper}

Paper questionnaires were handed to the patients during their visit to the outpatient clinic before and after surgery. The could use the prepaid envelope to return the questionnaires. When needed, patients were reminded by telephone to complete their questionnaire. The returned questionnaires were entered in an electronic database.

\section{Online follow-up}

Our software program (Research Manager ${ }^{\circledR}$ ) is an online database in which we prospectively collect all patient characteristics and surgical details. Patients received an automatically generated e-mail with a secured link to the online questionnaire, on set time points, based on the date of operation. Patients were reminded by email after seven days and more often, if needed. Patient responses are saved automatically in the online database. Preoperative details, surgical details and clinical details are filled in by the surgeon or ward physician.

All data are exportable to an Excel or SPSS file, which makes it easy to analyze for scientific purposes.

\section{Analyses}

For this study we selected all patients who underwent laparoscopic antireflux surgery in our clinic. We compared the response rate of a cohort of patients that used paper follow-up with a cohort that used online follow-up. Furthermore, we compared the completeness of the received questionnaires between the paper-collected and the onlinecollected data.

Few patients switched from paper follow-up to online follow-up. We performed a subanalysis with all switchers in which we compared the completeness of their questionnaires that were done on paper to the latter, completed online.

We compared baseline characteristics and results of the questionnaires between the paper and the online group. Furthermore, we analysed the improvement after surgery which we also compared between the paper group and the online group.

\section{Statistics}

Continuous data were analysed using normalcy tests to see which data were parametrically and non-parametrically distributed. Parametrically distributed data were analysed using Student $t$ tests. Non-parametric data were analysed using Mann-Whitney U tests. Categorical data were analysed using Chi-square tests and Fisher's exact tests (Figures 1 and 2). Statistical analyses were performed using IBM's Statistical Package for Social Sciences (SPSS), version 22 for Apple Macintosh OS (IBM corp., Armonk,New York, USA). A p value of less than 0.05 was considered to be statistically significant.

Preop: $\mathbf{n}=\mathbf{2 9 4}$

3 months: $n=271$

12 months: $n=219$

24 months: $n=140$

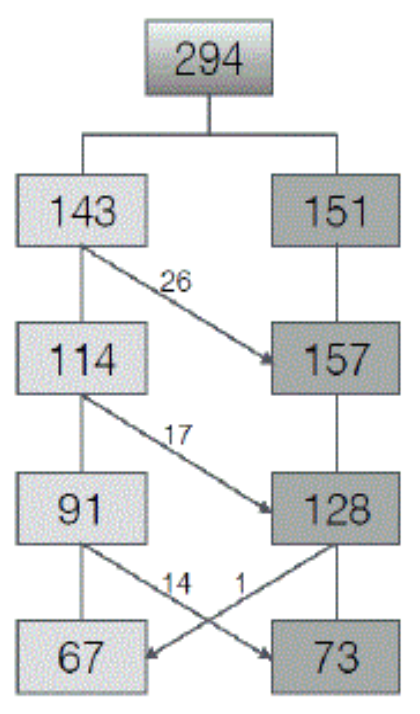

Figure 1. Flowchart. 


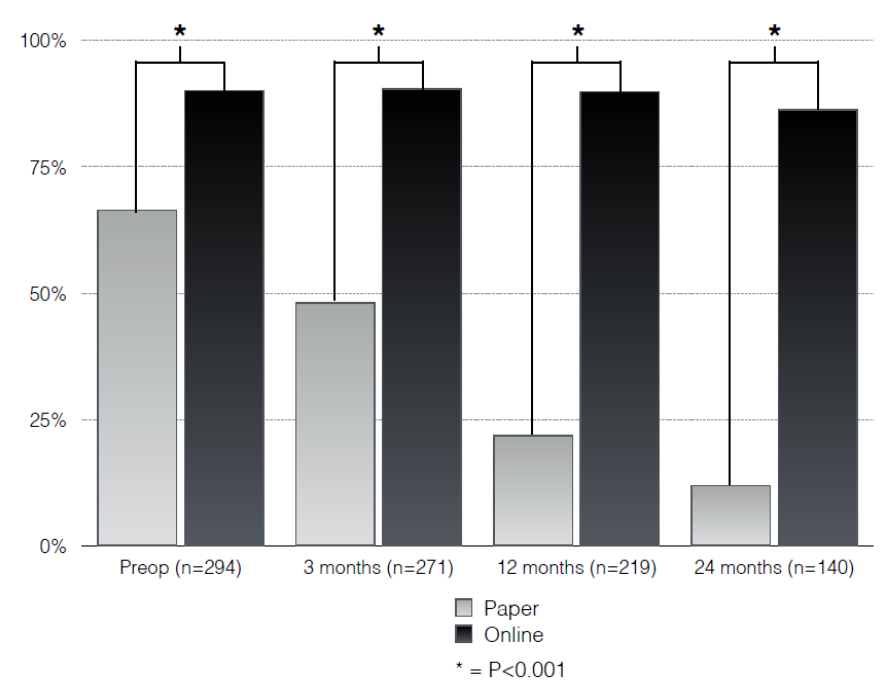

Figure 2. Response Rate.

\section{Ethical approval}

Patients gave informed consent and were informed about the purposes of the completed questionnaires and securely saved data. All data (both on paper and online) were coded before exported to the database. The Institutional Review Board of our hospital has evaluated our study protocol and approved it without further obligations.

\section{Results}

294 patients were included in our follow-up. Six patients were excluded (mentally incapability to fill in questionnaires $(n=2)$; age younger than 18 years $(n=1)$; incapability to speak and read the Dutch language $(n=1)$; blindness $(n=1)$; peroperative other diagnosis $(n=1)$ ). All patients underwent laparoscopic antireflux surgery, for either gastro-oesophageal reflux disease (GORD, $\mathrm{n}=148$ ), hiatal hernia oesophagei (HHO, $n=126)$ or achalasia $(n=20) .143$ patients started in the paper follow-up, 151 started in the online follow up. During follow-up in time, 57 patients switched from paper to online follow-up and one patient switched from online to paper follow-up (Figure 3). Patient characteristics were equal in both groups, except for operation type (Table 1). This was mainly because the number of Toupet fundoplications was higher in the online group due to participation in the MANTA trial as described above.

Response rates of the group using online follow up were significant higher compared to the group using follow-up on paper preoperatively, three months, one year and two years postoperatively $(\mathrm{P}<0.001$ (Figure 1)). The completeness of the collected data was significant better for the online group compared to the paper group $(\mathrm{P}<0.05$ (Figure 2)).

57 patients switched between follow-up method, from paper to online. Of these patients, $36(63.2 \%)$ responded their initial questionnaire on paper. Their next questionnaires were filled in using the online system. Subsequently, 50 of these 'switchers' (87.7\%) responded using the online system. Table 2 shows that the completeness of the received data for this group had increased significant with use of the online follow-up ( $77.8 \%$ on paper vs. $100 \%$ online; $\mathrm{P}=0.001)$.

At three months postoperative GERD-hr-QoL was lower in the paper group when compared to the online group (1.0 vs. $2.0 ; \mathrm{P}=0.046$ ). Preoperative and at 12 and 24 months postoperative the scores were equal. OES-24 scores were comparable in the paper and the online group. Satisfaction of surgery was better in the paper group at three months postoperative ( 9.0 vs. $8.0 ; \mathrm{P}=0.009$ ), but equal at 12 and 24 months postoperative. The majority of the patients would undergo the operation again, with the knowledge of what the effects were on their personal complaints. This is equal in the paper and the online group. Improvement of the GERD-hr-QoL and OES-24 was equal in the paper group and the online group (Table 3 ).

Table 1. Patient characteristics.

\begin{tabular}{|c|c|c|c|c|c|}
\hline & & $\begin{array}{l}\text { Overall } \\
(n=294)\end{array}$ & $\begin{array}{c}\text { Paper } \\
(n=143)\end{array}$ & $\begin{array}{c}\text { Online } \\
(n=151)\end{array}$ & P-value \\
\hline $\mathbf{A g e}^{\mathrm{a}}$ & & $56.3 \pm 15.3$ & $56.1 \pm 17.2$ & $56.5 \pm 13.3$ & 0.831 \\
\hline \multirow{2}{*}{ Gender $^{\mathrm{b}}$} & Male & $108(36.7 \%)$ & $48(33.6 \%)$ & $60(39.7 \%)$ & \multirow[t]{2}{*}{0.279} \\
\hline & Female & $186(63.3 \%)$ & $95(66.4 \%)$ & $91(60.3 \%)$ & \\
\hline Height $^{\mathrm{a}}$ & & $1.71 \pm 0.10$ & $1.71 \pm 0.10$ & $1.72 \pm 0.10$ & 0.244 \\
\hline Weight $^{\mathrm{a}}$ & & $81.2 \pm 13.9$ & $80.6 \pm 14.2$ & $81.7 \pm 13.7$ & 0.511 \\
\hline BMI $^{\mathbf{a}}$ & & $27.7 \pm 4.3$ & $27.7 \pm 4.6$ & $27.6 \pm 4.1$ & 0.804 \\
\hline \multirow[t]{7}{*}{ Pathology ${ }^{\mathrm{b}}$} & HHO & $126(42.9 \%)$ & $62(43.4 \%)$ & $64(42.7 \%)$ & \multirow{3}{*}{0.726} \\
\hline & GORD & $148(50.3 \%)$ & $73(51.0 \%)$ & $74(49.3 \%)$ & \\
\hline & Achalasia & $20(6.8 \%)$ & $8(5.6 \%)$ & $12(7.9 \%)$ & \\
\hline & $\begin{array}{c}\text { Anterior } \\
180^{\circ} \text { partial }\end{array}$ & $238(81.0 \%)$ & $118(82.5 \%)$ & $120(79.5 \%)$ & \multirow{4}{*}{$<0.001$} \\
\hline & Nissen & $19(6.5 \%)$ & $17(11.9 \%)$ & $2(1.3 \%)$ & \\
\hline & $\begin{array}{l}\text { Heller } \\
\text { myotomy \& } \\
\text { Dor }\end{array}$ & $20(6.8 \%)$ & $8(5.6 \%)$ & $12(7.9 \%)$ & \\
\hline & Toupet & $17(5.8 \%)$ & $0(0 \%)$ & $17(11.3 \%)$ & \\
\hline \multicolumn{2}{|c|}{ Re-operation ${ }^{\mathrm{b}}$} & $13(4.5 \%)$ & $8(5.6 \%)$ & $6(4.0 \%)$ & \multirow{2}{*}{0.653} \\
\hline \multicolumn{2}{|c|}{$\begin{array}{c}\text { Re-operation from other } \\
\text { clinic }^{b}\end{array}$} & $15(5.1 \%)$ & $6(4.2 \%)$ & $9(6.0 \%)$ & \\
\hline \multicolumn{6}{|c|}{$\begin{array}{l}\text { BMI = Body Mass Index } \\
\text { HHO = Hiatal Hernia Oesophagei; } \\
\text { GORD = Gastro-Oesophageal Reflux Disease Data presented as either a: mean (standard } \\
\text { deviation), or b: number (percentage). }\end{array}$} \\
\hline
\end{tabular}

Table 2. Completion rate of switchers between paper and online follow-up.

\begin{tabular}{|c|c|c|c|}
\hline & Initial paper $(\mathbf{n}=\mathbf{3 6})$ & Last online (n=50) & P-value \\
\hline Completion rate & $27(77.8 \%)$ & $50(100 \%)$ & 0.001 \\
\hline
\end{tabular}

Initial paper $=$ patients that completed their initial questionnaires on paper and switched to online during follow-up.

Last online $=$ patients that completed questionnaires online, while their previous questionnaires were completed on paper.

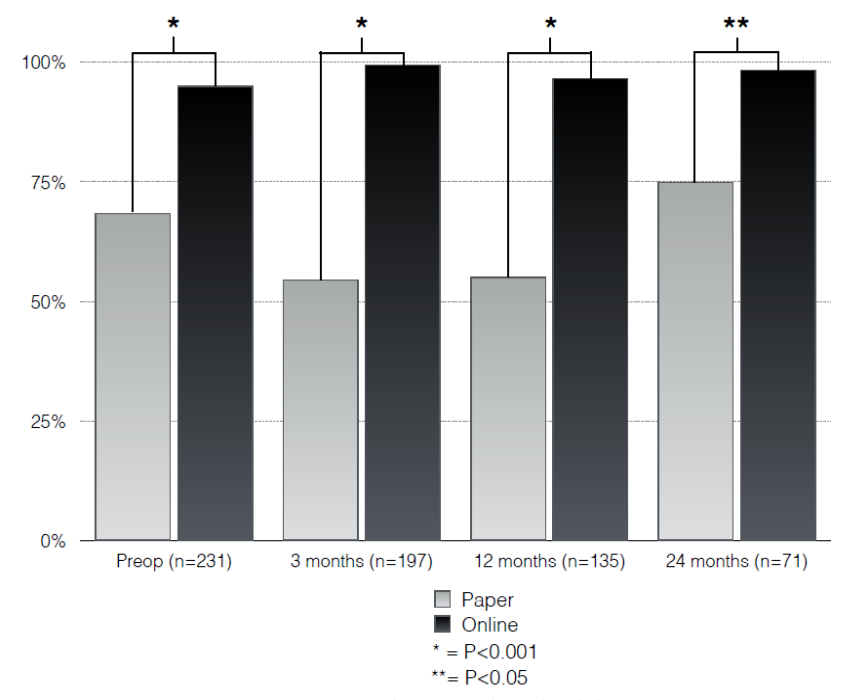

Figure 3. Completeness of received data. 
Table 3. Overall results of questionnaires

\begin{tabular}{|c|c|c|c|c|}
\hline & Preop & $\begin{array}{c}3 \text { months } \\
\text { postoperative }\end{array}$ & $\begin{array}{c}12 \text { months } \\
\text { postoperative }\end{array}$ & $\begin{array}{c}24 \text { months } \\
\text { postoperative }\end{array}$ \\
\hline GERD-hr-QoL ${ }^{a}$ & $13.0(13)^{*}$ & $1.0(5.0)^{* \dagger}$ & $2.0(7.0)^{* \dagger+}$ & $2.0(5.8)^{*+4}$ \\
\hline OES-24 & $46.0(14)^{*}$ & $36.0(11.0)^{* \dagger+}$ & $34.0(12.0)^{* \dagger+}$ & $33.0(11.0)^{* \dagger+}$ \\
\hline Satisfaction $^{\mathrm{a}}$ & - & $8.0(3.0)$ & $8.0(3.0)^{*}$ & $8.0(2.3)^{t}$ \\
\hline $\begin{array}{c}\text { Operation } \\
\text { again }^{\mathrm{b}}\end{array}$ & - & $175(90.7 \%)^{\ddagger}$ & $119(89.5 \%)^{\star}$ & $66(94.3 \%)^{\ddagger}$ \\
\hline
\end{tabular}

GERD-hr-QoL $=$ Gastro-oesophageal reflux disease health related quality of life questionnaire. OES-24 = dysphagia questionnaire.

Satisfaction $=$ satisfaction after surgery (VAS 1-10).

Operation again $=$ number of patients that would undergo the operation again, knowing the results afterwards.

Data presented as either a: median (interquartile range), or b: number (percentage). $*=$ significant difference when compared to preoperative scores $(\mathrm{P}<0.001) . \dagger=$ no difference in improvement when compared to preoperative scores between paper and online followup ( $\mathrm{P}>0.05)$. $\ddagger=$ equal in paper and online follow-up $(\mathrm{P}>0.05)$.

\section{Discussion}

Online follow-up leads to high completeness of data in this cohort following laparoscopic antireflux surgery. This is due to higher response rates, but also due to less missing data. Patients that initially start with paper follow-up show significant higher completeness of data when switching to online follow-up for their next questionnaires.

Patient Reported Outcome Measures (PROMs) are important to assess quality of care and outcome of treatments $(1-5,27)$. This can be measured with both validated questionnaires and Visual Analogue Scales, Likert-scores or Visick-scores [26,28,29].

In oncological surgery, PROMs are widely used. Registration of outcome is common and research with PROMs in cancer patients shows that its use enhances patient-clinician communication, patientsatisfaction and eventually quality of care [6-13,30-35]. Partly due to the lack of capacity or funding for traditional follow-up, long-term follow-up is generally not available for high-volume elective treatments such as cholecystectomy, inguinal hernia repair, rubber band ligation of hemorrhoids etc. Since National Health Agencies and insurance companies put more pressure on health care providers to warrant their results of treatment, it might be interesting to report those results [14]. Also, benign surgery is often performed to improve quality of life. Therefore, PROMs, and specifically health-related quality of life measures, are suitable for this follow-up.

In the past, several methods of collecting data were used, with different results. This varies from paper questionnaires sent by conventional mail, patient-reported diaries, telephone interviews, clinical or home interviews and to online, web-based questionnaires [36,37]. Electronic data collection methods were used as well, and showed to be reliable and valid with good patient participation [38-42]. Follow-up after surgery for benign diseases may ask for administrative and logistic challenges in a time with a lot of pressure on healthcare for budget cuts. Therefore, inventive and efficient methods of data collection with a low workload are needed. Russel et al found that an interactive automated telephone system was effective for a high response rate, and analysed the sociodemographic differences between patients that preferred different methods of data collections. In our cohort, availability of internet or computers was not an issue, so a socio-economic bias is not probable.

In this study baseline characteristics were comparable between the 'online' group and the 'offline' group (that completed follow-up on paper), except for the type of operation due to the multicenter MANTA-trial.
Our results show higher response rates and completeness of received data using online follow-up. Also, a group of switching patients show improvement in response rate and completeness of data with use of the online follow-up. This can be explained by the automated reminders for patients that did not complete their questionnaires, but also by the fact that it takes less effort for patients to complete their questionnaire. They can fill in the questionnaires on their smartphone and do not need to return the questionnaires during their next visit to the outpatient clinic nor have to bring the envelope to the mailbox. The higher response and completeness rates are also accompanied by low efforts for researchers, due to automated e-mails and automated reminders for patients.

Since a few patients favor follow-up on paper due to difficulties using the computer and accessing the internet, online follow-up should not be the only method offered to patients, and optional 'offline' followup on paper should still be available.

Postoperative improvement of scores were not different between the groups, which means the effects of antireflux surgery are comparable in the online and offline group. For the lower GERD-hr-QoL score three months postoperative for the paper follow-up group we do not have a good explanation. However, improvement of this score when compared to the preoperative score is comparable with the online follow-up. The OES-24 was the questionnaire that was most often incomplete. This can be explained by the length of this questionnaire: it is the section with the most questions.

Antireflux surgery has been overshadowed by bad results in the past, with poor follow-up. A strong belief that antireflux surgery should not be recommended to patients has dominated the referral pattern in the Netherlands (43). We chose to prospectively collect all data after antireflux surgery to provide objective and subjective highquality data for the referring physicians and for patients. The results of this follow-up can be used to rectify these thoughts, and restore the place of antireflux surgery as effective treatment. The patient-reportedoutcome-measures in this study can be used to adjust perioperative care and, if needed, surgical techniques, like it has done in the past (44). It is valuable for patients to present results directly derived from other patients, and to show the opinion of patients on success of their treatment. Instead of using common numbers from literature, this will lead to more precise data to inform patients about outcome, complications, PROMs and therefore patient satisfaction.

The data that are collected could be used for research purposes and health-standards, and for direct patient-care as well, since the results of the questionnaires can be directly accessed in the database during outpatient follow-up or other patient contacts.

The results of this study could be used to stimulate health professionals to create online data-collecting systems for follow-up of benign diseases. It could be widely used, with other options for more accurate and complete follow-up.

In conclusion, online follow-up leads to high response rates and high completeness of data. It is a user friendly way to collect data in large cohorts of patient with benign pathology. This data can be used to inform patients on the surgeon or hospital results for a specific treatment. Further studies for other treatments than antireflux surgery are warranted.

\section{Acknowledgements}

The authors thank Dr. Marco H. Blanker his advice on the revision of this manuscript. 


\section{Financial support}

There were no sources of outside support for research, including funding, equipment, and drugs.

\section{Conflicts of interest (real and potential)}

There are no real of potential conflicts of interest for all authors.

\section{Details of ethical approval}

The Institutional Research Board of our hospital has reviewed our study protocol and approved it without further obligations.

\section{References}

1. Patrick DL, Bergner M (1990) Measurement of health status in the 1990s. Annu Rev Public Health 11: 165-183. [Crossref]

2. Garratt A, Schmidt L, Mackintosh A, Fitzpatrick R (2002) Quality of life measurement: bibliographic study of patient assessed health outcome measures. BMJ 15:1417.

3. Marshall S, Haywood K, Fitzpatrick R (2006) Impact of patient-reported outcome measures on routine practice: a structured review. J Eval Clin Pract 12: 559-568.

4. Valderas JM, Alonso J (2008) Patient reported outcome measures: a model-based classification system for research and clinical practice. Qual Life Res 17:1125-1135.

5. FDA (2009) U.S. Department of Health and Human Services, Food and Drug Administration. Guidance for Industry. Patient-Reported Outcome Measures: Use in Medical Product Development to Support Labeling Claims.

6. van Gijn W, van de Velde CJ (2010) members of the EURECCA consortium. Improving quality of cancer care through surgical audit. Eur J Surg Oncol 36 Suppl 1: S23-S26.

7. Jakobsen E, Green A, Oesterlind K, Rasmussen TR, Iachina M, et al. (2013) Nationwide quality improvement in lung cancer care: the role of the Danish Lung Cancer Group and Registry. J Thorac Oncol 8: 1238-1247.

8. Van Leersum NJ, Snijders HS, Henneman D, Kolfschoten NE, Gooiker GA, et al. (2013) The Dutch surgical colorectal audit. Eur J Surg Oncol 39: 1063-1070. [Crossref]

9. de Steur WO, Henneman D, Allum WH, Dikken JL, van Sandick JW, et al. (2014) Common data items in seven European oesophagogastric cancer surgery registries: towards a European upper GI cancer audit (EURECCA Upper GI). Eur J Surg Oncol 40: 325-329.

10. Gaffney DK, Suneja G, Ryu SY, McCormick M, Plante M, et al. (2015) The Cervix Cancer Research Network: A Global Outreach Effort on Behalf of the Gynecologic Cancer InterGroup. Int J Radiat Oncol Biol Phys 92: 506-508.

11. van Dam PA, Tomatis M, Marotti L, Heil J, Wilson R, et al. (2015) The effect of EUSOMA certification on quality of breast cancer care. Eur J Surg Oncol 41: 14231429. [Crossref]

12. Govaert JA, van Dijk WA, Fiocco M, Scheffer AC, Gietelink L, et al. (2016) Nationwide Outcomes Measurement in Colorectal Cancer Surgery: Improving Quality and Reducing Costs. J Am Coll Surg 222: 19-29.e2.

13. Prades J, Manchon-Walsh P, Sola J, Espinas JA, Guarga A, et al. (2016) Improving clinical outcomes through centralization of rectal cancer surgery and clinical audit: a mixed-methods assessment. Eur J Public Health.

14. Conway PH, Mostashari F, Clancy C (2013) The future of quality measurement for improvement and accountability. JAMA 309: 2215-2216. [Crossref]

15. Gatenby PA, Bright T, Watson DI (2012) Anterior $180^{\circ}$ partial fundoplication--how I do it. J Gastrointest Surg 16: 2297-2303. [Crossref]

16. Jamieson GG, Watson DI, Britten-Jones R, Mitchell PC, Anvari M (1994) Laparoscopic Nissen fundoplication. Ann Surg 220: 137-145. [Crossref]

17. Toupet A (1963) Technic of esophago-gastroplasty with phrenogastropexy used in radical treatment of hiatal hernias as a supplement to Heller's operation in cardiospasms. Mem Acad Chir (Paris) 89: 384-389.

18. Patti MG, Arcerito M, De Pinto M, Feo CV, Tong J, et al. (1998) Comparison of thoracoscopic and laparoscopic Heller myotomy for achalasia. $J$ Gastrointest Surg 2: 561-566.

19. Rosati R, Fumagalli U, Bonavina L, Segalin A, Montorsi M, et al. (1995) Laparoscopic approach to esophageal achalasia. Am J Surg 169: 424-427. [Crossref]
20. Velanovich V, Vallance SR, Gusz JR, Tapia FV, Harkabus MA (1996) Quality of life scale for gastroesophageal reflux disease. J Am Coll Surg 183: 217-224. [Crossref]

21. Mouli VP, Ahuja V (2011) Questionnaire based gastroesophageal reflux disease (GERD) assessment scales. Indian J Gastroenterol 3: 108-117.

22. Rentz AM, Battista C, Trudeau E, Jones R, Robinson P, et al. (2001) Symptom and health-related quality-of-life measures for use in selected gastrointestinal disease studies: a review and synthesis of the literature. Pharmacoeconomics 19: 349-363.

23. Koetje JH, Nieuwenhuijs VB, Irvine T, Mayne GC, et al. (2016) Measuring Outcomes of Laparoscopic Anti-reflux Surgery: Quality of Life Versus Symptom Scores? World J Surg 40:1137-1144

24. Blazeby JM, Alderson D, Winstone K, Steyn R, Hammerlid E, et al. (1996) Development of an EORTC questionnaire module to be used in quality of life assessment for patients with oesophageal cancer. The EORTC Quality of Life Study Group. Eur J Cancer 32A: 1912-1917.

25. Viklund P, Lindblad M, Lagergren J (2005) Influence of surgery-related factors on quality of life after esophageal or cardia cancer resection. World J Surg 29: 841-848.

26. Grant S, Aitchison T, Henderson E, Christie J, Zare S, et al. (1999) A comparison of the reproducibility and the sensitivity to change of visual analogue scales, Borg scales, and Likert scales in normal subjects during submaximal exercise. Chest 116: 1208-1217.

27. Fitzpatrick R, Davey C, Buxton MJ, Jones DR (1998) Evaluating patient-based outcome measures for use in clinical trials. Health Technol Assess 2: 1-74.

28. Reips UD, Funke F (2008) Interval-level measurement with visual analogue scales in Internet-based research: VAS Generator. Behav Res Methods 40: 699-704.

29. Rijnhart-De Jong HG, Draaisma WA, Smout AJ, Broeders IA, Gooszen HG (2008) The Visick score: a good measure for the overall effect of antireflux surgery? Scand $J$ Gastroenterol 43: 787-793.

30. Bulman CH (1998) A ten year audit of the management of cancers of the larynx and pharynx. J Laryngol Otol 112: 948-953.

31. Detmar SB, Muller MJ, Schornagel JH, Wever LD, Aaronson NK (2002) Healthrelated quality-of-life assessments and patient-physician communication: a randomized controlled trial. JAMA 288: 3027-3034.

32. Velikova G, Booth L, Smith AB, Brown PM, Lynch P, et al. (2004) Measuring quality of life in routine oncology practice improves communication and patient well-being: a randomized controlled trial. J Clin Oncol 22: 714-724.

33. Simunovic M, Goldsmith C, Thabane L, McLeod R, Denardi F, et al. (2008) The Quality Initiative in Rectal Cancer (QIRC) trial: study protocol of a cluster randomized controlled trial in surgery. BMC Surg 8: 4-2482-8-4.

34. Jakobsen E, Palshof T, Osterlind K, Pilegaard H (2009) Data from a national lung cancer registry contributes to improve outcome and quality of surgery: Danish results. Eur J Cardiothorac Surg 35: 348-352.

35. Campbell I, Scott N, Seneviratne S, Kollias J, Walters D, et al. (2015) Breast cancer characteristics and survival differences between Maori, Pacific and other New Zealand women included in the Quality Audit program of Breast Surgeons of Australia and New Zealand. Asian Pac J Cancer Prev16: 2465-2472.

36. Weinberger M, Oddone EZ, Samsa GP, Landsman PB (1996) Are health-related quality-of-life measures affected by the mode of administration? J Clin Epidemiol 49 135-140. [Crossref]

37. Leidy NK, Elixhauser A, Rentz AM, Beach R, Pellock J, et al. (1999) Telephone validation of the Quality of Life in Epilepsy Inventory-89 (QOLIE-89). Epilepsia 40: 97-106. [Crossref]

38. Kleinman L, Leidy NK, Crawley J, Bonomi A, Schoenfeld P A comparative trial of paper-and-pencil versus computer administration of the Quality of Life in Reflux and Dyspepsia (QOLRAD) questionnaire. Med Care 39: 181-189.

39. Bushnell DM, Martin ML, Parasuraman B (2003) Electronic versus paper questionnaires: a further comparison in persons with asthma. J Asthma 40: 751-762.

40. Marks RG (2004) Validating electronic source data in clinical trials. Control Clin Trials 25: 437-446. [Crossref]

41. Bushnell DM, Reilly MC, Galani C, Martin ML, Ricci JF, et al. (2006) Validation of electronic data capture of the Irritable Bowel Syndrome--Quality of Life Measure, the Work Productivity and Activity Impairment Questionnaire for Irritable Bowel Syndrome and the EuroQol. Value Health 9: 98-105.

42. Welker JA (2007) Implementation of electronic data capture systems: barriers and solutions. Contemp Clin Trials 28: 329-336. [Crossref]

43. Boote MC (2006) Surgery rarely performed in reflux disease ("Zelden chirurgie bij antirefluxziekte"). Magma 12: 34-35. 
Jan H Koetje MD (2018) Online collection of Patient Reported Outcome Measures: an effective method for follow-up of benign surgery

44. Watson DI, Jamieson GG, Devitt PG, Mitchell PC, Game PA (1995) Paraoesophageal hiatus hernia: an important complication of laparoscopic Nissen fundoplication. $\mathrm{Br} J$ Surg 82: 521-523.
45. Koetje JH, Nieuwenhuijs VB, Irvine T, Mayne GC, et al. (2016) Measuring Outcomes of Laparoscopic Anti-reflux Surgery: Quality of Life Versus Symptom Scores? World J Surg 40: 1137-1144. [Crossref]

Copyright: (C2018 Jan H Koetje MD. This is an open-access article distributed under the terms of the Creative Commons Attribution License, which permits unrestricted use, distribution, and reproduction in any medium, provided the original author and source are credited. 